\section{Negociando fronteiras entre culturas, doenças e tratamentos no cotidiano familiar}

\author{
Negotiating the
} boundaries between cultures, illness, and treatments in everyday family life

Silvana Nair Leite

Professora do Curso de Farmácia da

Universidade do Vale do Itajaí

Rua Uruguai 458 - Centro

88302-202 Itajaí - SC - Brasil

$$
\text { snleite@univali.br }
$$

Maria da Penha Costa Vasconcellos

Professora da Faculdade de Saúde Pública da Universidade de São Paulo, Av. Dr. Arnaldo, 715

01246-904 São Paulo - SP - Brasil mpvascon@usp.br
LEITE, S. N.; VASCONCELLOS, M. P. C.: Negociando fronteiras entre culturas, doenças e tratamentos no cotidiano familiar. História, Ciências, Saúde - Manguinhos, v. 13, n. 1, p. 113-28, jan.-mar. 2006.

O texto propõe uma reflexão teórica acerca dos itinerários terapêuticos na prática familiar diária, considerando o uso dos mais diversos recursos no enfrentamento de episódios de doenças no cotidiano, tanto em ações isoladas, como nas interações sociais. A compreensão da doença enquanto fenômeno vivenciado não apenas pelo indivíduo, mas também no âmbito da sociedade, baseia a discussão das instâncias que afetam a procura por recursos terapêuticos, desde a escolha racional do indivíduo; a estrutura familiar enquanto provedora dos recursos básicos de saúde; as redes de relações sociais como fonte de apoio e informação nos cuidados de saúde, considerando as ações enquanto construtos sociais e não individuais; até as influências da cultura local e macro social, que fornecem para os grupos sociais as referências para interpretar as experiências e guiar suas ações. PALAVRAS-CHAVE: cultura; família; medicina popular.

LEITE, S. N.; VASCONCELLOS, M. P. C.: Negotiating the boundaries between cultures, illness, and treatments in everyday family life História, Ciências, Saúde - Manguinhos, v. 13, n. 1, p. 113-28, Jan.-Mar. 2006.

Viewing disease as a phenomenon experienced not only by the individual but also within the realm of society, the text is a theoretical reflection on the therapeutic methods ordinarily employed in household practice, which encompass a wide gamut of resources for responding to sickness. The factors affecting the search for treatment resources are discussed, including: individual rational choice, family structure as a provider of basic health resources, networks of social relations as a source of support and information on health care (considering initiatives as social rather than individual constructs), and the influence of the local and macro cultures, which provide social groups with references for interpreting their experiences and guiding their actions.

KEYWORDS: culture; family; popular medicine. 
A forma pela qual os indivíduos de uma dada sociedade situam-se em relação à doença, ou como a percebem, é tema reconhecido como de fundamental importância para o estudo social das doenças e das formas de lidar com elas. Desde tempos remotos, a humanidade teve de enfrentar a doença como parte de seu cotidiano, desenvolvendo, para tanto, estratégias diversas.

Longe de ser privilégio das ciências biomédicas e dos eruditos, os tratamentos das doenças são construções individuais e sociais que fazem parte da vida cotidiana em qualquer sociedade. Sendo o cotidiano um lugar privilegiado para o estudo da sociedade e suas transformações, lugar onde se configuram e atuam os "pequenos jogos sociais que constituem a trama social", conforme Pais (2003, p. 75), é, pois, no cotidiano que se encontra o cenário de base para o estudo dos itinerários terapêuticos.

Desta forma, o que se pretende aqui é realizar uma reflexão teórica acerca dos itinerários terapêuticos, tomando o enfrentamento aos episódios de doença como parte constitutiva do cotidiano, ou daquilo que o perturba.

O trabalho de campo deste projeto, que constituiu extensão dessa reflexão, privilegiou durante, aproximadamente, nove meses, o uso dos mais diversos recursos terapêuticos na prática familiar diária, considerando os episódios de doenças no contexto da vida familiar, tanto em ações isoladas, como nas interações sociais. Busca-se, assim, compreender os processos de escolha que desenham os itinerários terapêuticos, e não apenas descrever os caminhos seguidos pelos indivíduos ou, como é objetivo de alguns estudos, basear a questão da terapêutica na utilização de determinados serviços de saúde. É nesse sentido que se vai visualizar o campo temático, de tal modo a refletir sobre ele teórica e metodologicamente.

Ao apontar para as terapêuticas, entendidas aqui como qualquer forma de tratamento de doenças, é imprescindível delimitar o que entendemos por 'tratável', ou o que entendemos por 'doença'. Feita esta contextualização, vamos nos voltar para a estrutura familiar, as redes sociais, a cultura local e macro-social e, também neste projeto, para o indivíduo.

\section{Que doença?}

Alves e Rabelo (1999) afirmam que a doença é a experiência de mal-estar, de que algo não vai bem, não necessariamente um problema biológico, o que pontua o caráter pessoal e particular dessa experiência: “ $\mathrm{O}$ 'mal-estar' torna-se doença quando é transformado em objeto socialmente aceito de conhecimento e intervenção. É real porque se origina no mundo do senso comum" (Alves \& Rabelo, 1999, p. 173). Para Helman (1994, p. 104), que concorda com isso, “a doença é a resposta subjetiva do paciente, e de todos os que o 
cercam, ao seu mal-estar". Compreender a doença significa, pois, estender o olhar sobre o sujeito e sobre o campo de interações entre indivíduos, grupos e instituições e a cultura subjacente.

A importância das diferentes dimensões da doença pode ser expressa pelas diferentes designações encontradas na literatura sobre o tema. A "enfermidade (disease) é o que o órgão tem, a doença (illness) é o que o doente tem" (Helman, 1994, p. 104). Podemos acrescentar a idéia de que se considera doente (sickness) aquele que o meio, a sociedade vê como doente. Eisenberg (in Uchoa \& Vidal, 1994) compactua com a mesma visão ao definir a "doença processo" e a "doença experiência". A primeira refere-se às anormalidades de estrutura ou funcionamento de órgãos ou sistemas, e a segunda, à experiência subjetiva de mal-estar sentido pelo doente. A doença, tal como é percebida pelo doente, não se refere apenas ao "processo patológico no sentido biomédico do termo. Considera-se que ela conjuga normas, valores e expectativas, tanto individuais como coletivas, e se expressa sob formas específicas de pensar e agir" (Uchoa \& Vidal, 1994, p. 500). Alves (1993) vai além, ao tratar a doença como uma experiência intersubjetiva, construída pela experiência própria do indivíduo através de processos concretos de interação social.

"A definição de doença não inclui somente a experiência pessoal do problema de saúde, mas também o significado que o indivíduo confere a ela." Os mesmos sintomas ou enfermidades podem ser interpretados de maneiras completamente diferentes por indivíduos de culturas diferentes ou em contextos diferentes (Helman, 1994, p. 104). A doença está, portanto, intimamente relacionada à cultura. As doenças acabam por simbolizar grande parte das ansiedades mais comuns das pessoas ou da sociedade, os perigos da vida cotidiana. Para Figueira (in Rocha, 2000), a saúde e a forma de reconhecer e tratar a doença estão diretamente relaciuonadas à visão de mundo do sujeito.

A forma como um indivíduo conceitua um episódio de doençae isso inclui etiologia, curso e duração dos sintomas, diagnóstico e formas de tratamento - é designada como modelo explanatório por Pachter (1994). Esses processos são experimentados por indivíduos, mas influenciados em grande parte por crenças, atitudes e valores culturalmente construídos, congregando sistemas referenciais diferentes, tanto populares como científicos. Para o autor, são muito raros os modelos explanatórios exclusivamente populares ou exclusivamente científicos, uma vez que a compreensão da doença inclui, necessariamente, o campo da interação de saberes, valores e expectativas.

Como fenômeno social, "a desordem é sempre interpretada pelo doente, pelo médico e pelas famílias" (Kleinman \& Good, in Uchoa \& Vidal, 1994, p. 500). Neste campo é interessante observar, tam- 
bém, a existência das chamadas 'doenças populares', que Rubel (in Helman, 1994, p. 109) definiu como "síndromes das quais os membros de determinado grupo dizem ser vítimas e para as quais sua cultura reconhece uma etiologia, um diagnóstico, medidas preventivas e sistemas de cura". Em outras palavras, trata-se do mal-estar reconhecido por aquela sociedade como doença. Aqui, vale salientar que, quando se busca estudar as terapêuticas no âmbito popular, é imprescindível considerar essas 'doenças populares' enquanto entidades reais e merecedoras de atenção e, mais ainda, que representam episódios importantes para a compreensão do tema saúde/ doença/tratamento naquela comunidade.

A doença, portanto, é um tema extremamente complexo e passível de diferentes leituras que vão da sociologia à medicina, tratando quer de seu caráter individual, fisiológico, quer de seu aspecto cultural ou social. Da mesma forma, são diversas as compreensões dos modos de lidar com a doença, da procura por serviços, das estratégias formuladas no dia-a-dia, para seu enfrentamento.

A terapêtica: para onde olhar?

Estima-se que somente 10 a 30 por cento dos sintomas percebidos recebem atenção médica. (Haajer-Ruskamp \& Hemminki, 1993). Isto é motivo de grande preocupação por parte dos setores de saúde, pelo risco que a falta de atenção médica representa para a vida, além de constituir-se em fato muito explorado pela indústria de medicamentos; o que nos leva a perguntar como se processa todo o conjunto dos cuidados dos sintomas percebidos. Algumas tentativas de compreensão nessa área dão respostas pontuais, tais como, "doença leve pode ser tratada em casa, doença grave é que precisa de médico" (Loyola, 1982, p. 168). Quando se trata de doenças autolimitadas, são resolvidas sem atendimento médico (HaaijerRuskamp \& Hemminki, 1993), ou, simplesmente, pelo serviço de saúde, caso exista. Estas formas de descrever os itinerários terapêuticos são, com certeza, demasiadamente reduzidas e não se aproximam nem mesmo das expectativas que os profissionais da área da saúde e os das ciências sociais têm sobre a compreensão do uso que a população faz dos recursos terapêuticos.

Os estudos realmente dedicados ao entendimento do tema refletem a complexidade da compreensão da própria doença e das diferentes teorias sobre a ação, em especial as ações relativas à saúde. As abordagens variam desde a concepção das escolhas terapêuticas realizadas pelos indivíduos como produto de operações cognitivas, racionais, até a interpretação macrossocial das ações individuais em casos de doença, passando por influências culturais e das redes de relações sociais, a começar pelo suporte social básico, a família. 


\section{a) A família}

Helman (1994) descreve a família como a primeira arena de assistência informal. Entre 70 e 90 por cento dos tratamentos de saúde ocorrem na família. Para M. Andréia Loyola (1982), nas classes populares, primeiramente esgotam-se as possibilidades de recursos terapêuticos familiares, advindos de experiências acumuladas que permitem medir a eficácia, tanto de uma prática propriamente popular, como de especialistas. Nas enfermidades infantis, por exemplo, o papel da mãe ou cuidadora é decisivo sobre os cuidados de saúde. Boltanski (1989) afirma que a maioria das doenças infantis são tidas como normais da idade e tratadas em casa, pela mãe; quando 'mal curadas' ou quando o conjunto dos sintomas se aproxima do que as mães consideram grave é que buscam o médico. Na tentativa de compreender as escolhas por diferentes tratamentos, Boltanski descreve que as mães fazem duas operações mentais, quando detectam algo errado com as crianças: relacionam os sintomas percebidos com o rol de doenças conhecidas e, então, relacionam a doença com o rol de remédios indicados para curar a referida doença.

Menéndez (1992) também descreve a família como primeira instância de cuidados de saúde, acrescentando que ela, ou o grupo doméstico, constitui a unidade onde se constroem e operam os principais determinantes da morbidade e mortalidade nos diferentes grupos etários, particularmente dos menores de cinco anos, tanto positiva quanto negativamente. É também a instituição sobre a qual repercutem as conseqüências das crises econômicas. Para o autor, não cabe dúvida de que a família, ou grupo doméstico, é uma das unidades de descrição e análise do processo saúde/doença/ atenção mais estratégicas, pelo papel central que ela desempenha em tal processo:

O sujeito, ao pensar um padecimento ou uma enfermidade, ao decidir que fazer com ela, não atua isoladamente e sim como parte de uma micro/macro estrutura. A família constitui a micro estrutura que mais peso teria na constituição das representações e práticas dos sujeitos em relação ao processo saúde/doença/ atenção. (Menéndez, 1992, p. 5)

O mesmo autor argumenta que, apesar de se tratar de uma unidade importante, a família muitas vezes é representada em sua cultura, seus hábitos e modo de vida pela condução da mulher-mãe, categoria decisiva para as ações de saúde no lar. Caponi (2000) analisa o papel da mãe como representante das demandas médico-higienistas no lar no transcurso do século XIX, e o papel da unidade 'família' para a normalização da sociedade ao modelo emergente na época. A família e a mãe podem ser consideradas, então, 'recursos' para o 
setor saúde, como sugere Menéndez (1992), pois são utilizadas para aplicar programas de educação em saúde, de planejamento familiar, de higiene e tantos outros. Na prática cotidiana, as mães, e suas famílias, utilizam-se das informações dos setores biomédicos e populares para a constituição de sua estrutura de apoio na sobrevivência do grupo.

\section{b) As redes de relações sociais}

A influência inegável da família enquanto grupo mais restrito nos processos de decisão terapêutica, não se sustenta como forma isolada de compreender esses processos, contribuindo mais no âmbito de observação. A família 'ampliada', ou a rede de relações sociais mediada por ela ou pelos próprios indivíduos, tem sido tomada por diversos autores como objeto de estudo mais apropriado para a compreensão das formas de lidar com a doença e as escolhas terapêuticas.

O termo 'rede social', segundo Barbosa et al. (2000, p. 6) "refere-se ao conjunto de pessoas em uma população e suas conexões. A análise de redes sociais baseia-se no pressuposto da importância das relações entre unidades que interagem, isto é, relações definidas como ligações entre unidades constituem componente fundamental das teorias de redes". Criados a partir de modelos matemáticos de arranjos entre pontos e linhas, os estudos das redes sociais normalmente são focados na análise da estrutura das redes, ficando o estudo das interações menos desenvolvido.

Pescosolido (1992) argumenta que todo indivíduo é um ser social e, por isso, o foco de análise deve ser o indivíduo em suas interações sociais. Para a autora, "os indivíduos nem são fantoches de alguma estrutura abstrata nem são calculistas individualistas", como se encontram em algumas teorias; para a autora, "as pessoas modelam e são modeladas por redes de relações sociais". Ela propõe uma estrutura para análise dos cuidados de saúde que chamou de estratégia de organização social, baseada em análise de episódios concretos e redes de relações sociais.

Claramente delineada pela interpretação das ações enquanto construtos sociais e não individuais, a proposta de se olhar para as interações sociais na busca de compreender os cuidados de saúde é bem justificada na própria compreensão mais corrente da doença, enquanto fenômeno de dimensão social, como exposto anteriormente. O próprio reconhecimento da ocorrência de uma doença é compartilhado com a sociedade, assim como o papel de enfermo, a legitimação da doença e das formas de tratá-la. Reconhecer que as interações sociais são fundamentais para a compreensão dos cuidados de saúde, sem dúvida, é uma grande contribuição dos estudos de redes sociais e saúde. 
No entanto, alguns autores têm chamado a atenção para a demasiada circunscrição observada nos estudos de redes sociais em torno das estruturas das redes como definidores dos padrões de comportamento esperados dos indivíduos a elas ligados. Souza (1999) discute várias questões sobre a utilidade e aplicabilidade desses modelos. Entre as questões levantadas aponta a excessiva limitação dos estudos que se referem à correlação entre estrutura da rede e a procura, ou não, por serviços médicos 'legais' (biomedicina). Ao focar os cuidados de saúde na procura por médicos, argumenta a autora, "ignora-se que a trajetória percorrida pelo enfermo, desde o momento em que a situação começa a se mostrar problemática até a busca de ajuda institucional (médica ou não), não é absolutamente linear", ignorando as diversas estratégias desenvolvidas nesse percurso, derivadas de experimentação ou de hábitos culturalmente adquiridos.

Estudando as estratégias alimentares entre famílias pobres, Gerhardt (2003, p. 724) defende que "é a dinâmica das relações humanas que está na base da estrutura das redes e das formas de entre-ajuda", e que esse conceito de 'redes' não pode ser compreendido separadamente do contexto em que se constituem, incluindo relações afetivas, companheirismo e solidariedade. Tem-se, portanto, o campo das relações e interações entre os indivíduos como objeto a ser considerado no estudo dos itinerários terapêuticos, porém com o cuidado de não negligenciar outros campos de observação, como o dos hábitos e crenças culturalmente adquiridos que, como se pode observar nos exemplos a seguir, compõe uma interface com a influência das redes de relações sociais.

\section{c) A dimensão cultural dos cuidados de saúde}

Diversos estudos têm demonstrado como as peculiaridades de crenças e costumes interferem no modo como os cuidadores tratam de questões relacionadas à saúde das crianças, como vacinação, alimentação, cuidados puerpérios e amamentação. No sul do Brasil, Béria et al. (1993) identificaram crenças que influenciaram o padrão de prescrição médica e a forma como as mães de crianças com diarréia tratavam o problema. Até mesmo a vida de crianças é determinada por costumes em povos mais isolados, e o diagnóstico da causa mortis é influenciado por tal tradição (Allotey \& Reidpath, 2001).

Uchoa \& Vidal (1994) citam trabalhos que sugerem relação entre as concepções culturais das doenças e a utilização de recursos terapêuticos ou métodos de prevenção. Um desses trabalhos, de Agyepong (1992), mostra que, para certos grupos de Gana, a causa da malária não está relacionada ao vetor e sim ao calor, inviabilizando os métodos de prevenção, e que o tratamento com cloroquina não é reconhecido como eficaz porque a doença reapa- 
rece em outro período, por isso é pouco utilizado. Citando Geertz (1978), os autores argumentam que a "cultura como universo de símbolos e significados permite aos indivíduos de um grupo interpretar a experiência e guiar suas ações"; trata-se do contexto no qual os diferentes eventos tornam-se inteligíveis (Uchoa \& Vidal, 1994, p. 500), concordando com Alves \& Rabelo (1999), ao afirmarem que as respostas aos problemas criados pelas doenças constituem-se socialmente e remetem diretamente a um mundo compartilhado de práticas, crenças e valores. Tal premissa remete ao relato de Lévi-Strauss sobre a eficácia dos tratamentos do xamã. Diz o autor que "a mitologia do xamã não corresponde a uma realidade objetiva, não tem importância: o doente acredita nela, e ele é membro de uma sociedade que acredita" (1996, p. 228); descreve o que chama de "complexo xamanístico", formado pelo xamã que executa seu ritual, pelo doente que experimenta a cura e pelo público, que também participa da cura.

A visão de mundo do indivíduo, reflexo da cultura em que está inserido, é utilizada por Rocha (2000) para o embasamento de seu estudo sobre as trajetórias terapêuticas de pacientes de serviços psiquiátricos. Refere-se à proposta de Figueira (1978), em que este descreve os sistemas de compreensão do mundo aos quais as pessoas estão vinculadas e os estilos possíveis entre as pessoas em relação à terapêutica em uma sociedade complexa. O sujeito poderia optar pelo isolamento, quando é totalmente orientado por um único sistema simbólico; pela relativização, se tem uma visão de mundo fundamental, mas convive com outras, que considera viável; ou pela desorientação, quando o sujeito se sente impotente frente à pluralidade de visões de mundo e circula entre elas à procura da terapêutica mais eficiente, não se mantendo fiel a nenhuma delas. Rocha, entretanto, conclui que, para os sujeitos, não há oposição ou inviabilidade entre sistemas terapêuticos diferentes, como psiquiatria e práticas espirituais, e sim complementaridade, sem que um enfraqueça a importância do outro.

Mesmo concluindo que, para os sujeitos, não há oposição entre os sistemas terapêuticos, Rocha faz referência à percepção dos sujeitos das diferentes explicações etiológicas que resultam nos diferentes sistemas terapêuticos. Possíveis reflexos da força da cultura biomédica no contexto atual, os modelos etiológico-terapêuticos são também descritos por Laplantine (1991) ao analisar a literatura, entrevistar médicos e pacientes. O autor apresenta quatro modelos etiológicos e quatro modelos terapêuticos entre todas as arenas pesquisadas. Gorter et al. (1995) demonstrou que o diagnóstico que a mãe faz em casos de diarréia (se é compreendido como empacho, sol de vista, caída de la mollera) é muito importante na hora de decidir o tipo de ajuda e o tratamento que cada caso requer. Dessa forma, o autor descreve, no contexto dos parâmetros biomédicos, 
os conhecimentos e hábitos típicos de um determinado sistema cultural que reconhece as doenças citadas.

Esta nítida aproximação entre a compreensão dos hábitos e conhecimentos culturalmente adquiridos e a relação causa-tratamento é contestada por alguns autores. Helman (1994, p. 70), ao analisar os diferentes sistemas terapêuticos disponíveis em uma sociedade, diz que "para o indivíduo doente, a origem desses tratamentos importa menos do que sua eficácia em aliviar o sofrimento". No estudo de Boltanski (1989, p. 22) entre diferentes classes sociais nos arredores de Paris, descrevem-se as ações terapêuticas populares, aquilo que o autor chamou de uma 'medicina imitativa' por não haver preocupação com a origem ou as causas das doenças, ou com um conjunto de princípios que torne possível sua explicação: "é suficiente saber reconhecer a gripe e saber que se trata com Antigripine. Diversos estudiosos do social já indicaram que muitas práticas do ser humano são constituídas por hábitos construídos na rotina cultural, nas ações do dia-a-dia (Pescosolido, 1992), e passam a integrar o cotidiano sem contestações, sem por isso deixar de representar importante campo de estudo e, como lembra Pais (2003), sem restringir a vida cotidiana a estas práticas habituais 'inconscientes'.

Entretanto, apesar das discordâncias sobre a importância ou não da percepção de causas bem definidas para a escolha dos tratamentos, e da constatação de que muitas práticas advêm do hábito, é imprescindível retornar aos estudos antropológicos que indicam que a visão de mundo que norteia o indivíduo em determinada sociedade - as crenças e costumes que fazem parte de sua cultura estabelece um 'fazer sentido' que interfere no processo saúde/doença/tratamento. Voltando aos estudos de Lévi-Strauss (1996, p. 228) sobre as curas xamanísticas, vale lembrar o relato, muito sugestivo, da intervenção de um curandeiro no caso de um parto:

os espíritos protetores e os espíritos malfazejos, os monstros sobrenaturais e os animais mágicos, fazem parte de um sistema coerente que fundamenta a concepção indígena do universo. A doente os aceita, ou, mais exatamente, ela não os pôs jamais em dúvida. O que ela não aceita são dores incoerentes e arbitrárias, que constituem um elemento estranho a seu sistema, mas que, por apelo ao mito, o xamã vai reintegrar num conjunto onde todos os elementos se apóiam mutuamente e tendo compreendido, não se resigna, apenas, ela sara.

A cultura fornece os elementos para que os eventos, as práticas do cotidiano, inclusive as relativas à saúde, sejam compreendidas e aceitas ou, para citar Geertz, "sejam inteligíveis". Reis e Fradique (2003) chamam a atenção para o fato de que antes do encontro com o técnico, a pessoa já construiu uma narrativa pessoal sobre o seu 
problema de saúde ou sobre uma determinada atitude ou ação preventiva. Essas expectativas que o paciente traz sobre a doença e o tratamento podem estar diretamente relacionadas com a aceitação da terapia, pois indicam as interpretações que o paciente já formulou sobre o problema, que pode coincidir ou não com o que o médico propõe. Para Gonçalves et al. (1999), "obedecer à prescrição dos medicamentos é, além de concretizar a doença, efetivar as suas limitações", pois reafirma sua incapacidade de compreender o tratamento e o que está acontecendo consigo, é aceitar uma imposição que muitas vezes não lhe faz sentido.

Nesse sentido, Helman (1994) compreende que as escolhas que as pessoas fazem entre diferentes tipos de curandeiros (chama de curandeiro todo agente de cura, sejam eles médicos, rezadores, benzedores, farmacêuticos e outros) não são definitivas ou incontestáveis, pois elas também escolhem entre os diagnósticos e as recomendações que 'fazem sentido'. Esta questão é percebida por alguns autores como uma justificativa para a sobrevivência, o que inclui até o crescente uso de terapias consideradas tradicionais, antigas ou populares, ao contrário de um entendimento muito comum de que a falta de recursos médicos modernos seria a causa da sobrevivência de tais formas de terapia. A reflexão de Laplantine é ilustrativa desta questão. Para ele,

enquanto a intervenção médica oficial pretende apenas fornecer uma explicação experimental dos mecanismos químico-biológicos da morbidez e dos meios eficazes para controlá-los, as medicinas populares associam uma resposta integral a uma série de insatisfações (não apenas somáticas, mas psicológicas, sociais, espirituais para alguns, existenciais para todos) que o racionalismo social não se mostra disposto a eliminar. (Laplantine, 1991, p. 220)

Ao optar por um curandeiro popular, o indivíduo estaria optando por uma explicação sobre a doença que lhe é inteligível, com termos e conceitos sobre saúde, sobre a causa da doença, sobre o tratamento necessário e sobre o próprio corpo que condizem com a sua percepção, ou que, ao menos, ele possa compreender e aceitar. Assim, como exemplifica Boltanski (1989), o curandeiro popular explica ao doente que sua dor é originária de estômago caído, que caiu porque o corpo dilatou, e que o tratamento consiste em enfaixar a região para suspender o órgão. A cultura, desta forma, fornece uma maneira de entendimento do corpo e do seu funcionamento, e o indivíduo utiliza esse conhecimento para organizar as informações disponíveis e dar-lhes coerência. Uma operação racional, portanto.

O 'fazer sentido' é, pois, a congruência entre a base cultural do sujeito e o processo por ele desenvolvido para uma ação de saúde. Esse processo de escolha individual baseia as teorias cognitivas que procuram explicar os variados itinerários terapêuticos. 


\section{d) $O$ processo individual de escolha terapêutica}

Boltanski (1989), ao relatar as práticas das mães nos cuidados de saúde dos seus filhos, utiliza uma explicação para as escolhas que elas fazem. Essa explicação centra-se em uma seqüência de operações mentais utilizando como base os conhecimentos adquiridos no cotidiano. Com exceção dos hábitos incorporados à vida cotidiana que não estejam diretamente relacionados com a racionalidade, todas as crenças, conhecimentos, valores construídos e transmitidos no âmbito cultural também estão envolvidos na prática diária com as chamadas estruturas cognitivas dos indivíduos, com um 'racionalizar' sobre os recursos disponíveis para fazer as escolhas.

Vários autores defendem que as escolhas terapêuticas que os indivíduos fazem são frutos de operações racionais, baseadas em uma lógica econômica, de avaliação de custo-benefício. Alves e Rabelo (1999) citam os estudos iniciados por Mechanic e Volkart, em 1960, e outros autores, que tinham uma "forte coloração pragmática: os indivíduos orientam racionalmente a conduta para a satisfação das suas necessidades", os indivíduos estariam aptos, portanto, a avaliar as possibilidades disponíveis no mercado e optarem por aquela que traria maiores vantagens. Pescosolido (1992) cita o trabalho de Lindenberg, de 1985, para o qual "o ponto teórico inicial é o indivíduo que, tendo preferências e confrontando-se com dificuldades, tem que fazer escolhas".

Sob essa concepção, a unidade de análise é o indivíduo. Aí, procuram-se 'explicações' em uma lógica dedutiva, considerando as ações do dia-a-dia e, neste caso, especificamente, as ações relativas à saúde, dentro de uma racionalização econômica, de custo-benefício, da maior vantagem. Essa lógica funcionalista está presente em diversos estudos sobre utilização de recursos terapêuticos, como nos estudos de adesão à terapêutica. Alguns dos fatores mais facilmente relacionados com a não-adesão (de maneira especial os estatisticamente relacionados) são de caráter prático, como ausência de sintomas, maior número de doses/dia, o alto custo dos produtos, a ocorrência de efeitos colaterais (Leite \& Vasconcellos, 2003). Alguns estudos fundados em abordagem qualitativa sugerem que a questão da adesão deve ser enfocada pela perspectiva do usuário enquanto sujeito de suas ações. Conrad (1985) descreve uma forma de 'teste' que portadores de epilepsia desenvolvem como prática medicamentosa para avaliar o progresso da doença, para controlar a dependência aos medicamentos e diminuir o estigma de 'doente' que os medicamentos representam. Para o autor, os pacientes procuram obter um controle sobre o seu estado através do controle do uso de medicamentos, uma perspectiva, portanto, racionalizada e de caráter individual sobre o uso de medicamentos. Dowell e 
Hudson (1997) também estudaram o tema pela perspectiva do usuário e descrevem um "modelo de decisão terapêutica". Eles explicam as diferentes atitudes dos pacientes frente ao uso de medicamentos prescritos pelos tipos psicológicos: os céticos, os usuários passivos e os usuários ativos, estes, os que representam o modelo racionalista para empreender ações de saúde, pesando efeitos colaterais, possível dependência, grau de gravidade da doença, a aceitação da própria condição de doente.

Esse tipo de explicação racionalista das ações dos indivíduos nos cuidados de saúde reflete o modelo de sociedade capitalista vigente: individualista, que prega a responsabilidade individual sobre a vida com base na suposta liberdade individual. A tal influência todos estamos submetidos, como podemos observar em grande parte dos estudos que, embora hoje não tomem essa abordagem como única, consideram tal racionalização econômica em suas análises. Se a influência recai sobre as linhas de interpretação, também não se pode negar que qualquer indivíduo está sendo influenciado por esse modelo de sociedade e pelas formas de nela sobreviver e, então, a lógica racionalista pode mesmo, nos tempos atuais, fazer parte das práticas diárias dos sujeitos.

Autores como Pescosolido (1992) têm criticado essa abordagem que toma o indivíduo como unidade de análise e que utiliza a perspectiva racionalista para explicar os processos de escolha terapêutica. Essa autora defende que somente tomando o indivíduo em sua rede de relações sociais pode-se compreender as ações por ele tomadas, pois a abordagem individualista encara o indivíduo como ser egoísta, racional, mas deixa escapar fenômenos permeados por incertezas, por ignorância, acaso, inconsciência.

Os modelos explicativos descritos pelo grupo de Kleinman (1981) comparam valores da cultura subjacente a determinados grupos, como o dos profissionais de saúde e o dos usuários, que formam a base para as explicações que racionalizam as ações de saúde em cada grupo. Alves (1993) pontua que os modelos explicativos de Kleinman não podem dar conta da compreensão da experiência da enfermidade, pois "as percepções, crenças e ações dos indivíduos são geralmente heterogêneas, complexas e ambíguas", e que os conhecimentos e práticas dos indivíduos são construídos de e por experiências diversas, em situações biográficas determinadas. Para Alves, a experiência da enfermidade, como também as ações relacionadas a ela, têm caráter intersubjetivo, integrando interpretações subjetivas dos indivíduos aos processos de interação social permeados por certos padrões culturais. 


\section{Os itinerários terapêuticos: um amplo campo a ser explorado}

A literatura registra diversos modos de olhar para a doença, diversas interpretações sobre o sentir-se doente e sobre o uso de recursos terapêuticos, alguns dos quais já foram aqui mencionados. De modo geral, há uma tendência em construir 'modelos' com os quais é possível explicar as escolhas que os indivíduos fazem sobre terapêutica: os modelos etiológico-terapêuticos ou de relação causa-tratamento, o modelo de decisão terapêutica, o modelo de crenças de saúde, os modelos explicativos, e as justificativas eminentemente práticas, baseadas em ações do cotidiano. A maior parte dessas abordagens sugere que o sujeito, ao identificar uma doença, opta por este ou aquele recurso terapêutico, e o faz de forma racionalizada, calculada, sustentado por diversas concepções e influências.

Se considerarmos que os estudos estão, de alguma forma, abordando os itinerários terapêuticos, uma vez que procuram explicar as ações relativas a partes ou ao conjunto dos usos de recursos terapêuticos em uma sociedade, podemos questionar: quais desses modelos podem ser adotados como "verdadeiros modelos, além das clivagens regionais", como sugere Laplantine (1991), "que consistem na atualização de formas elementares da doença e da cura que possam ser apontados em qualquer sociedade"? Quando pensamos em itinerário, pensamos em um conjunto de planos, estratégias, projetos desenvolvidos pelos indivíduos com o objetivo de enfrentar o problema, mas será que os itinerários são planejados como uma seqüência de ações? E os hábitos, o acaso, os atos impensados, não constituem parte dos itinerários terapêuticos? A tendência é sempre a de buscar uma lógica explicativa, racional, por isso a busca constante pela construção de modelos teóricos que possam colocar esse conjunto (não necessariamente um plano) de ações em uma esquadria.

Nesse sentido é interessante anotar o alerta de Alves e Souza (1999, p. 131): “o itinerário terapêutico é um fenômeno por demais complexo para que possa ser subsumido a generalidades que procedem pela descoberta de leis que ordenam o social". Os autores argumentam que a restrição das condutas humanas tende a simplificar as ações e os próprios atores sociais a "modelos tipológicos ou classificatórios predeterminados".

Com a proposta de estudar os itinerários terapêuticos torna-se necessário procurar propostas metodológicas e teorias sociais mais amplas e flexíveis que possibilitem ao pesquisador ampliar seu olhar e sua percepção. O objeto de estudo requer essa flexibilidade do pesquisador, como justificam Sevalho e Castiel (1998, p. 48-9): 
quanto ao adoecer humano, é inevitável encará-lo como um objeto indisciplinado que resiste a nossas tentativas disciplinares de enquadramento. Somente assim, acreditamos ser possível transitar por doenças nas populações e moléstias e suas representações nos grupos sócio-culturais, assumindo os riscos de nos perdermos pelos (des)caminhos indisciplinares, em busca de compreensão para intervenções menos insatisfatórias do que as prevalentes no campo biomédico-epidemiológico atual.

Considerando a 'indisciplinaridade' do objeto, mas também as interpretações resultantes dos diversos olhares já lançados sobre ele, podemos sugerir que o itinerário terapêutico (caminho, trajetória em busca da cura) envolve ações de distintas origens e finalidades. Se considerarmos que a sociedade atual está baseada na lógica custo-benefício, e que muitas das ações empreendidas no cotidiano são permeadas por essa lógica, temos de considerar que a lógica econômica, em termos biomédicos, a que os profissionais de saúde foram aculturados não corresponde necessariamente à lógica popular. Com todo o conhecimento sobre a diversidade e complexidade do social produzido pelos estudos antropológicos nas últimas décadas, é improvável pensar que exista um modelo ou padrão dentro do qual poderemos enquadrar os processos de escolha. Ainda nem podemos afirmar que todas as terapêuticas adotadas no nível popular são decorrentes de um 'processo de escolha', porém é muito significativo o 'fazer sentido' para o indivíduo, e esta pode ser a forma de racionalização empreendida: a lógica permeada por conhecimentos e hábitos culturais, muitos dos quais sem justificativa aparente, mas que constroem a forma como o mundo é entendido pelo indivíduo e, mais que isto, negociado nas relações sociais e legitimado pela sociedade.

A complexidade que envolve a vida cotidiana e o enfrentamento das doenças em geral não faz parte da formação do profissional de saúde, e a diferença substancial entre os elementos que alimentam o raciocínio desses profissionais e os diversos grupos populares

* Texto elaborado a partir de Projeto de Tese de Doutorado "Recursos terapêuticos adotados no âmbito familiar para

tratamento de crianças de 0-5 anos em ItajaíSC", do Departamento de Saúde Materno Infantil da Faculdade de Saúde Pública da Universidade de São Paulo. Apoio Financeiro: Organização

Panamericana de Saúde, Capes, Univali. proporciona grande dificuldade para que o profissional compreenda as atitudes de seus pacientes, resultando em relações distanciadas e preconceituosas. Em uma realidade econômico-social como a que vive o nosso país e a maior parte da população mundial, parece muito coerente que as terapias populares e tradicionais sejam incorporadas aos sistemas de saúde, como propôs a Organização Mundial de Saúde em 1978. Muitas ações nesse sentido, no entanto, tentam entender e racionalizar as práticas populares de saúde dentro da lógica biomédica, descontextualizando e desvirtuando o seu sentido. Ainda estamos um passo atrás, perseguindo a compreensão da utilização dos recursos terapêuticos pelos indivíduos, desde a 'simples' procura por serviços médicos ou adesão à terapia prescrita até a utilização de terapias populares. 
Não há dúvida de que, diante de tantas interrogações, as incertezas sobre o complexo campo do enfrentamento das doenças devem permear os estudos sobre o tema e a formação, sempre tão afirmativa, dos profissionais de saúde.

\section{REFERÊNCIAS BIBLIOGRÁFICAS}

Allotey, P.; Reidpath, D. Establishing the causes of childhood mortality in Ghana: the spirit child. 2001

Social Science and Medicine, v. 52, p. 1007-12.

Agyepong, I. A. 1992

Malaria ethnomedical perceptions and practice in an Adangbe farming community and implications for control. Social Sciences and Medicine, v. 35, p. 131-7.

Alves, P C

A experiência da enfermidade: considerações teóricas.

Cadernos de Saúde Pública, v. 9, n. 3, p. 263-71.

Alves, P. C. B. \& Rabelo, M. C. M.

Significação e metáforas na experiência da enfermidade.

In: Rabelo, M. C. M.; Alves, P. C. B. \& Souza, I. M. A.

Experiência de doença e narrativa. Rio de Janeiro: Fiocruz. p. 171-86.

Alves, P. C. B. \& Souza, I. M. A.

Escolha e avaliação de tratamento para problemas de saúde: considerações sobre o itinerário terapêutico. In: Rabelo, M. C. M.; Alves, P. C. B. \& Souza, I. M. A. Experiência de doença e narrativa. Rio de Janeiro: Fiocruz. p. 125-36.

Barbosa, M. T. S.;

Byintong, M. R. L.;

Struchiner, C. J.

2000

Béria, J. U. et al. 1993

Boltanski, L. 1989

Caponi, S.

Conrad, P. 1985

Modelos dinâmicos e redes sociais: revisão e reflexões a respeito de sua contribuição para o entendimento da epidemia do HIV.

Cadernos de Saúde Pública, v. 16, supl. 1.

Epidemiologia do consumo de medicamentos em crianças de centro urbano da região sul do Brasil. Revista de Saúde Pública, v. 27, p. 95-104.

As classes sociais e o corpo.

São Paulo: Graal.

Da compaixão à solidariedade: uma genealogia da assistência médica. Rio de Janeiro: Fiocruz.

The meaning of medications: another look at compliance. Social Science and Medicine, v. 20, n. 1, p. 29-37.

Dowell, J.; Hudson, $\mathrm{H}$. 1997

Figueira, S. A. 1978

Geertz, C. 1978

Gerhardt, T. E. 2003

Gonçalves, $\mathrm{H}$. et al. 1999

Gorter, A. C. et al. 1995

A qualitative study of medication-taking behaviour in primary care. Family Practice, v. 14, n. 5, p. 369-75.

Notas Introdutórias ao Estudo das Terapêuticas II: Robert Castel e Michel Foucault. Rio de Janeiro: Editora Campus.

A interpretação das culturas.

Rio de Janeiro: Zahar.

Situações de vida, pobreza e saúde: estratégias alimentares e práticas sociais no meio urbano. Ciência E Saúde Coletiva, v. 8, n. 3, p. 713-26.

Adesão à terapêutica da tuberculose em Pelotas, RS, na perspectiva do paciente. Cadernos de Saúde Pública, v. 15, n. 4.

Diarrea infantile en la Nicaragua rural: creencias y prácticas de salud tradicionales. Bol Oficina Sanitaria Panamericana (OPS), v. 119, n. 5, p. 377-90. 
Haaijer-Ruskamp, F. M.; Hemminki, E. 1993

Helman, C. G. 1994

Kleinman, A. 1981

Laplantine, F. 1991

Leite, S. N.;

Vasconcellos, M. P. 2003

Lévi-Strauss, C. 1996

Loyola, M. A. 1984

Menéndez, E. L. 1992

Organização Mundial da Saúde 1978

Pachter, L. M. 1994

Pais, J. M. 2003

Pescosolido, B. A. 1992

Reis, J. C.;

Fradique, F. S. 2003

Rocha, R. M. 2000

Sevalho, G.;

Castiel, L. D. 1998

Souza, I. M. A. 1999

Uchoa, E.; Vidal, J. M. 1994
The social aspects of drug use. In: Dukes, M. N. G. Drug utilization studies. Copenhagen: WHO Regional Office for Europe.

Cultura, saúde e doença.

Porto Alegre: Artes médicas.

Patients and Healers in the context of cultures.

Berkeley: University of California.

Antropologia da doença.

São Paulo: Matins Fontes.

Adesão à terapia medicamentosa: elementos para a discussão de conceitos e pressupostos adotados na literatura. Ciência E Saúde Coletiva, v. 8, n. 3 , p. $775-82$.

Antropologia estrutural.

Rio de Janeiro: Tempo brasileiro.

Médicos e curandeiros: conflito social e saúde.

Rio de Janeiro: Difel.

Grupo doméstico y proceso salud/enfermedad/atención.

Del 'teoricismo' al movimiento continuo.

Cuadernos Medico Sociales, v. 59, p. 3-18.

Atencion primaria de salud: informe de la Conferencia Internacional sobre Atención Primaria e Salud. Alma-Ata: WHO.

Culture and clinical care.

Journal of American Medical Association, v. 271, n. 9, p. 690-4.

Vida cotidiana: enigmas e revelações.

São Paulo: Cortez.

Beyond rational choice: the social dynamics of how people seek help. American Journal of Sociology, v. 97, n. 4, p. 1096-138.

Significações sobre causas e prevenção das doenças em jovens adultos, adultos de meia-idade e idosos.

Psicologia: teoria e pesquisa, v. 19, n. 1 .

Trajetórias terapêuticas de usuários de serviços psiquiátricos e adeptos de umbanda: um estudo sobre pluralismo terapêutico.

Revista de Enfermagem UERJ, v. 8, n. 2, p. 73-7.

Epidemiologia e antropologia médica: a possível in(ter)disciplinaridade. In: Alves, P. C.; Rabelo, M. C. (org.) Antropologia da saúde: traçando identidade e explorando fronteiras. Rio de Janeiro: Relume Dumará. p. 47-70.

$\mathrm{Na}$ trama da doença: uma discussão sobre redes sociais e doença mental. In: Rabelo, M. C. M.; Alves, P. C. B. \& Souza, I. M. A Experiência de doença e narrativa. Rio de Janeiro: Fiocruz. p. 89-124.

Antropologia médica: elementos conceituais e metodológicos para uma abordagem da saúde e da doença. Cadernos de Saúde Pública, v. 10, n. 4 , p. $497-504$. 